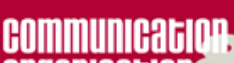
Organlofidin.

\section{Communication et organisation}

Revue scientifique francophone en Communication organisationnelle

$57 \mid 2020$

La question européenne dans les organisations

\title{
ABDELNOUR Sarah et MÉDA Dominique (dir.), 2019. Les nouveaux travailleurs des applis
}

Paris : Presses Universitaires de France. La vie des idées.

ISBN 978-2-13-081523-5

\section{Carole Fagadé}

\section{(2) OpenEdition}

\section{Journals}

Édition électronique

URL : https://journals.openedition.org/communicationorganisation/9103

DOI : 10.4000/communicationorganisation.9103

ISSN : $1775-3546$

Éditeur

Presses universitaires de Bordeaux

Édition imprimée

Date de publication : 1 juin 2020

Pagination : 199-201

ISSN : 1168-5549

\section{Référence électronique}

Carole Fagadé, «ABDELNOUR Sarah et MÉDA Dominique (dir.), 2019. Les nouveaux travailleurs des applis », Communication et organisation [En ligne], 57 | 2020, mis en ligne le 01 juin 2020, consulté le 24 mars 2023. URL : http://journals.openedition.org/communicationorganisation/9103 ; DOI : https:// doi.org/10.4000/communicationorganisation.9103 
Eloria Vigouroux-Zugasti est docteure en sciences de l'information, et de la communication, A.T.E.R. à l'Université Paris-Est Marne-La-Vallée, chercheure associée au laboratoire MICA (EA 4426) et au laboratoire DICEN-IDF (EA 7339).

\section{ABDELNOUR Sarah et MÉDA Dominique (dir.), 2019. Les nouveaux travailleurs des applis}

Paris : Presses Universitaires de France. La vie des idées

\section{Par Carole Fagadé}

Les nouveaux travailleurs des applis est un ouvrage dirigé par Sarah Abdelnour et Dominique Méda. L'objectif des contributeurs est d'étudier le concept de " capitalisme de plateforme ", qui désigne l'" externalisation des travailleurs et le déplacement des frontières du travail mais aussi la création de valeur et son partage, inégalitaire, entre d'une part, les détenteurs des algorithmes, sites et applications que sont les plateformes et, d'autre part, les travailleurs présents sur celles-ci » (p. 10).

Un certain nombre de questions relatives à ce concept ont suscité l'intérêt des chercheurs et chercheuses en sociologie, en sciences politiques et en droit qui ont participé à l'élaboration de cet ouvrage. Ces questions concernent la structuration sociale, le déplacement des frontières entre travail professionnel, travail domestique et travail bénévole et, enfin, la marchandisation du travail d' "à côté ". Les analyses présentées dans cet ouvrage portent également sur la " neutralité » des plateformes numériques qui se présentent comme des intermédiaires facilitant la mise en relation des utilisateurs. C'est à travers des entretiens menés exclusivement avec des travailleurs des plateformes de services en ligne et de vente d'objets, la réalisation de plusieurs enquêtes quantitatives et l'analyse de données collectées sur des sites internet que les contributeurs du présent ouvrage tentent de répondre à ces différentes questions.

Tout d'abord, dans son chapitre sur le rapport entre le " capitalisme de plateforme ", l' "économie collaborative " et l'« économie du partage », Diane Rodet affirme que l'économie de plateforme n'a jamais été collaborative. L'économie de plateforme se rapproche davantage de l' "économie du chiffre d'affaires » (p. 20). En effet, les plateformes numériques « ne relèvent pas (...) d'une économie du partage au sens où il s'agirait de donner à autrui une partie de ce que l'on possède " (p. 20). Si les promoteurs du capitalisme de plateforme attribuent à ce système un "fort " potentiel en écologie et en lien social, l'auteure explique qu'il convient de distinguer le « capitalisme de plateforme » de l'Économie Sociale et Solidaire (ESS) qui repose 
sur un modèle non-marchand.

De plus, les analyses proposées par Pauline Barraud de Lagerie et Luc Sigalo Santos sur les plateformes de microtravail et sur le crowdworking révèlent la similarité de ces plateformes avec le tâcheronnat ou le sweating system du $19^{\mathrm{e}}$ siècle. En effet, les activités effectuées sur les plateformes de microtravail sont associées à " un travail à la tâche, non qualifié, réalisé chez soi, pour un salaire de misère " (p. 40). Le " tâcheronnat " constitue, selon les auteurs, un système d'intermédiaires qui tirent profit de la soustraitance et de la capacité dont ils disposent à faire "suer " les ouvriers.

Concernant la marchandisation, Anne Jourdain et Sidonie Naulin ont observé des blogs, des plateformes de vente d'objets et des plateformes de vente de services. Elles dévoilent non seulement l'émergence de nouvelles tâches, de nouvelles compétences et de nouveaux modes d'organisation mais aussi le pouvoir détenu par les plateformes de fixer la valeur économique des tâches à effectuer : « l'existence du travail produite par l'économie numérique tient moins à la création de nouveaux emplois et à la démocratisation du marché du travail qu'à la transformation des contenus même du travail et non à son intensification» (p. 60).

Enfin, en se focalisant sur les livreurs à vélo et les Véhicules de Transport avec Chauffeur (VTC), Sarah Abdelnour et Sophie Bernard étudient les résistances collectives, ainsi que les conditions pouvant favoriser leur émergence, face au capitalisme de plateforme. Les résultats de leur enquête soulignent un double préjudice à l'égard des chauffeurs grévistes victimes de la dégradation de leurs conditions de travail d'une part et, d'autre part, de "l'injustice " ressentie face à leurs pairs nongrévistes qui profitent de la grève pour faire des " courses rentables" (p. 67). Ce chapitre questionne le caractère passionné, libre, autonome et bien rémunéré que l'on a attribué au travail ces dernières années.

Dans leur contribution, Hélène Nasom-Tissandier et Morgan Sweeney s'intéressent à la question de la neutralité des plateformes numériques de transport quant aux obligations juridiques liées aux prestations des travailleurs. En effet, face aux contentieux, les travailleurs des plateformes de transport sont principalement confrontés à la non-reconnaissance de leur statut de travailleur indépendant. Les auteurs suggèrent d'outrepasser la neutralité des plateformes de services numériques et d'envisager l'attribution de responsabilités, en octroyant à ces plateformes le statut d'entreprise ou d'employeur. Ceci se justifie, d'un côté, par le fait que le travailleur ne possède pas d'entreprise et, de l'autre, par la présence d'un lien de subordination " classique " dans l'exercice du travail de plateforme.

En guise de conclusion, l'ouvrage tente de montrer la nécessité d'une désillusion. Cette dernière partie commentée par Dominique Méda incite le lecteur à rompre avec l'« illusion romantique » d'une possible autonomie idéalisée qui existerait en 
dehors du salariat. Elle recommande au contraire de se focaliser sur le renforcement de l'attractivité du salariat, en améliorant non seulement les conditions de travail mais aussi l'autonomie des salariés. La lecture de cet ouvrage dirigé par Sarah Abdelnour et Dominique Méda intéressera tous ceux qui sont intéressés par l'économie du numérique en général et par les travailleurs des plateformes numériques en particulier.

\section{Référence}

ABDELNOUR Sarah et MÉDA Dominique (dir.), 2019. Les nouveaux travailleurs des applis. Paris : Presses Universitaires de France. La vie des idées. ISBN 978-213-081523-5, 9,50€.

Carole Fagadé prépare sa thèse en Sciences de l'Information et de la Communication à l'Université Bordeaux Montaigne (MICA EA 4426). Elle étudie l'intégration des dispositifs numériques d'information et de communication dans les universités béninoises. Elle s'intéresse précisément aux facteurs socio-culturels, organisationnels et scolaires qui participent à l'appropriation de la technologie mobile chez les scolaires.

DENIS, Jérôme, 2018. Le travail invisible des données : éléments pour une sociologie des infrastructures scripturales

Paris : Presses des Mines

\section{Par Élise Lehoux}

"Décidément, on ne devrait jamais parler de "données" mais “d'obtenues" ». Cette citation de Bruno Latour ouvre le dernier chapitre de l'ouvrage de Jérôme Denis, Le travail invisible des données. Eléments pour une sociologie des infrastructures scripturales, dont la pensée nourrit cet essai très stimulant. Elle met en exergue l'un des objectifs de cette enquête, visant à restituer « la face cachée des données » (p. 17).

Jérôme Denis introduit son propos par un exemple tiré de sa propre expérience qui lui a permis de prendre conscience des modalités de " fabrication " des données, à l'occasion du décès de son père. Il s'attache à décrire le parcours des données dans un contexte de deuil : les différentes étapes de la production, les activités de lecture et d'écriture, la complexité des opérations informationnelles qui ont permis $\mathrm{d}$ ' " actualiser ", progressivement, la mort de son père face aux différentes institutions. Les données deviennent dans ce contexte « agissantes » (p. 19). 